\title{
Adult Wilms' tumor with calcification untreated for 5 years - a case
} report

\author{
Hiroshi Masuda1,2, Haruhito Azuma*2, Fumitaka Nakajima², \\ Toshikazu Watsuji ${ }^{1}$ and Yoji Katsuoka ${ }^{2}$
}

Address: ${ }^{1}$ Department of Urology, Hirakata City Hospital, Hirakata, Osaka 569-8686, Japan and 2Department of Urology, Osaka Medical College, Takatsuki, Osaka 569-8686, Japan

Email: Hiroshi Masuda -m3255makiko@r2.dion.ne.jp; Haruhito Azuma* - uro004@poh.osaka-med.ac.jp; Fumitaka Nakajima - uro007@poh.osaka-med.ac.jp; Toshikazu Watsuji - hhp08-watsuji@kcat.zaq.ne.jp; Yoji Katsuoka - uro001@poh.osakamed.ac.jp

* Corresponding author

Published: 05 June 2004

BMC Urology 2004, 4:5
Received: 06 February 2004

Accepted: 05 June 2004

This article is available from: http://www.biomedcentral.com/l47I-2490/4/5

(C) 2004 Masuda et al; licensee BioMed Central Ltd. This is an Open Access article: verbatim copying and redistribution of this article are permitted in all media for any purpose, provided this notice is preserved along with the article's original URL.

\begin{abstract}
Background: Wilms' tumor is rarely found in adults and there are no established treatment guidelines for such tumors in adults. Whereas calcification is a common finding in neuroblastoma, it is considered uncommon in Wilms' tumor.

Case presentation: We report a case of adult Wilms' tumor with calcification in a 22-year-old man. He had been initially referred to our hospital with a chief complaint of right flank pain 5 years previously, when abdominal computed tomography had revealed focal calcification at the upper pole of the right kidney. Although we planned further assessment, he did not revisit our hospital again until 5 years later, again because of right flank pain. Ultrasound and computed tomography scan revealed a large mass lesion with calcification in the right kidney, invasive to the hepatic lobe. The patient underwent curative right nephrectomy and partial right hepatic lobectomy. Pathological analysis demonstrated a nephroblastoma (Wilms' tumor) with predominant epithelial histology infiltrating the hepatic lobe. The patient has been well without tumor recurrence for 15 months after surgery.
\end{abstract}

Conclusions: Calcification may be a sign of slow tumor gowth and possibly a favorable prognosis in cases of adult Wilms' tumor.

\section{Background}

Wilms' tumor mostly occurs in childhood, and there are no established treatment guidelines for such tumors in adults. Whereas calcification is a common finding in cases of neuroblastoma, it is considered uncommon in Wilms' tumor. Here we describe a case of adult Wilms' tumor with calcification in a 22-year-old man, who was untreated for 5 years but made a good recovery without disease progression after surgery. Calcification may be a sign of slow tumor growth and possibly a favorable prognosis in cases of Wilms' tumor in adults. 

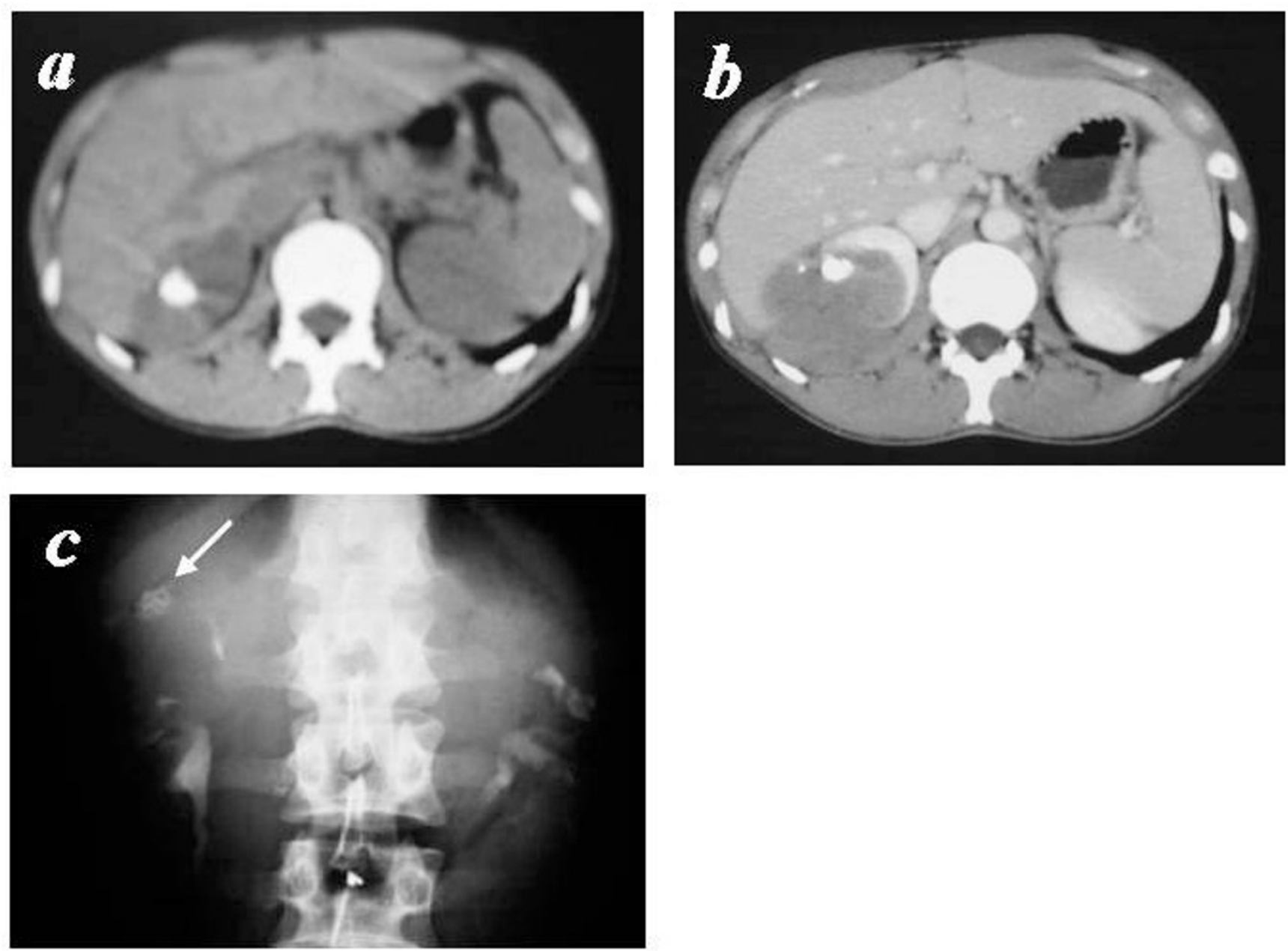

\section{Figure I}

Abdominal computed tomography scans taken at the first (a) and second (b) visits, and drip infusion pyelography conducted at the second visit (c) a) Focal calcification at the upper pole of the right kidney. b) A large right renal mass with calcification, invasive to the hepatic lobe. c) Focal calcification at the upper pole of the right kidney (white arrow).

\section{Case presentation}

A 17-year-old man with a chief complaint of right flank pain was referred to our hospital. Abdominal computed tomography (CT) revealed focal calcification at the upper pole of the right kidney (Fig. 1-a). We suspected renal stones in a caliceal diverticulum, and planned further assessment including drip infusion pyelography (DIP). However, because the patient became asymptomatic, he made no further visits to our hospital for 5 years. At the age of 22 years, however, he presented again because of right flank pain. DIP demonstrated focal calcification at the upper pole of the right kidney, and ultrasound (US) and CT scan demonstrated a large right renal mass with calcification invading the hepatic lobe (Figs. 1-b, and 1-c). Selective renal arterial angiography demonstrated a hypovascular tumor in the same area where the mass lesion had been observed by US, DIP, and CT. The patient underwent curative right nephrectomy and partial right hepatic lobectomy under a diagnosis of malignant renal tumor. The upper pole of the extirpated kidney was occupied by the tumor, which adhered tightly to the liver. The mass was completely removed along with the hepatic lobe, without rupture. No other invasion or lymph node metastasis was found. Macroscopic examination showed an encapsulated tumor with calcification that had infiltrated hepatic lobe (Fig. 2). The specimen weighed 280.0 g. The cut surface was solid and homogeneously whiteyellow. Pathological analysis demonstrated a nephroblastoma (Wilms' tumor) with a predominantly epithelial histology that had infiltrated the hepatic lobe. The disease 


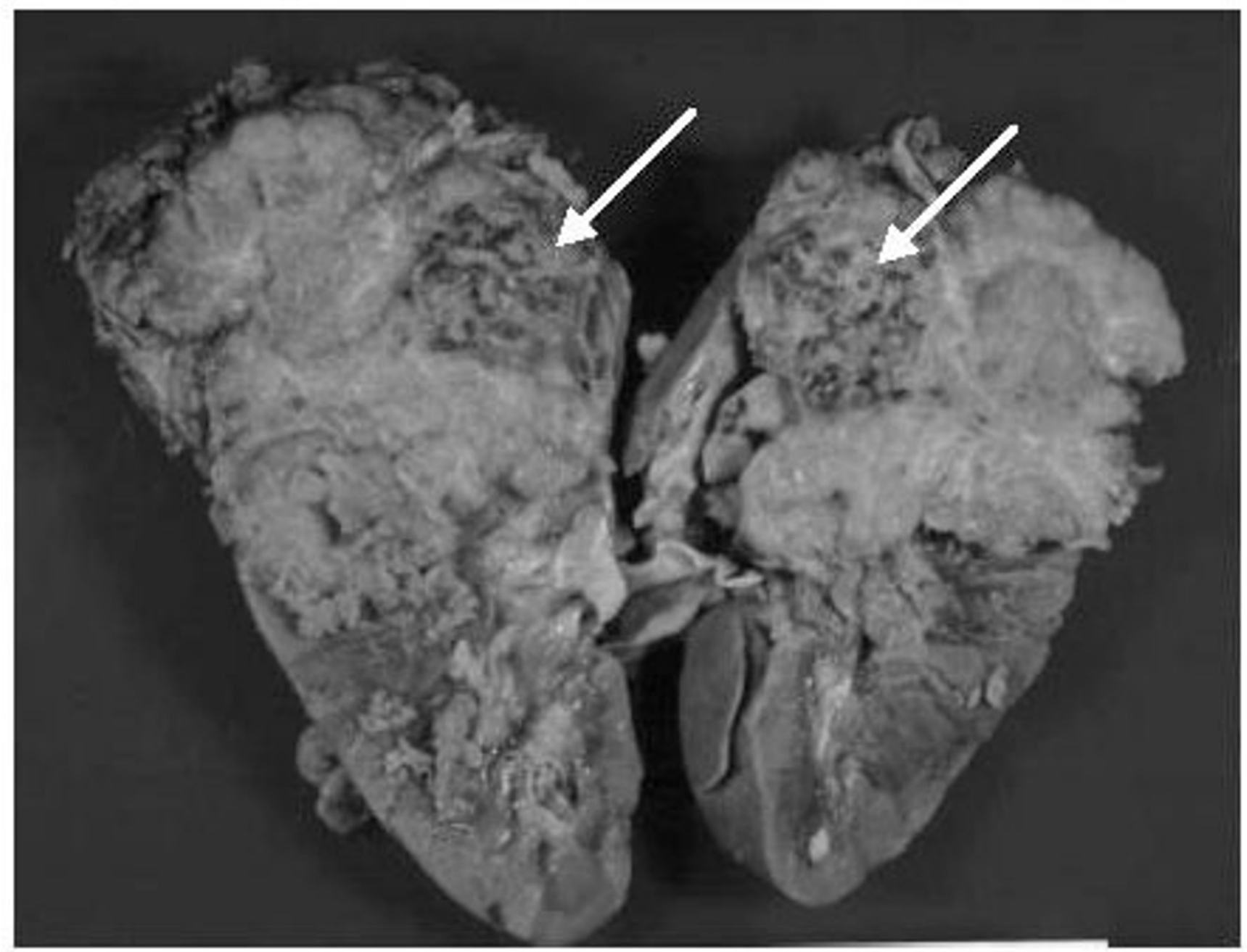

Figure 2

Macroscopic appearance of the tumor The tumor is encapsulated with calcification (arrows), and shows invasion to the hepatic lobe.

was classified as stage II according to the National Wilms' Tumor Study classification. After surgery, the patient received adjuvant chemotherapy with vincristine-actinomycin D for 60 weeks. He has been well without tumor recurrence for 15 months after surgery.

\section{Discussion}

Wilms' tumor is rare in adults, whereas it is the most common renal tumor in children. Adult Wilms' tumor may have a more aggressive clinical course and a higher tumor stage at the time of diagnosis compared with that in children [1]. In the present case, the patient presented with a chief complaint of right flank pain, and abdominal CT revealed focal calcification at the upper pole of the right kidney. We suspected renal stones in a caliceal diverticu- lum, and planned further assessment including DIP, but the patient did not visit our hospital again for a further 5 years, by which time his clinical stage had progressed to stage II from stage I at the time of initial diagnosis, according to the National Wilms' Tumor Study classification. Intratumoral calcification is a common finding in neuroblastoma, but rare in Wilms' tumor [2]. The incidence of radiographically demonstrable calcification in primary Wilms' tumor varies between $3 \%$ and $17 \%$ [3]. It has not been clarified whether there is a significant relationship between tumor calcification and histology, grade, or patient prognosis. However, several previous reports have demonstrated that although calcified tumors may be relatively large, they tend to be localized and histologically well differentiated. This may indicate that calcified tumors 
are slow-growing and that calcification in Wilms' tumor may be a sign of a favorable prognosis [4]. In the present case, the 5-year delay in treatment was unfortunate because this allowed the tumor to become large and invade the liver. However, the patient has been well without tumor recurrence for 15 months after radical surgery. This may also support the concept that calcified Wilms' tumors in adults show a low degree of aggressiveness.

\section{Conclusions}

Calcification in adult Wilms' tumor may be a sign of slow tumor growth and possibly a favorable prognosis.

\section{References}

I. Adolphs HD, Knopfel G, Vogel J: Wilms' tumor in the adolescent and adult. Eur J Urol I983, 9:28I-287.

2. Phillips TL, Chin FG, Palubinskas AJ: Calcification in renal masses: an eleven-year survey. Radiology 1963, 80:786-794.

3. Kaufaman RA, Holt JF, Heidelberger KP: Calcification in primary and metastatic Wilms' tumor. Am J Roentgenol 1978, 130:783-785.

4. Krieger JN, Sinderman KW, Seligson GR: Calcified renal cell carcinoma: a clinical, radiographic and pathologic study. J Urol 1979, I 21:575-580.

\section{Pre-publication history}

The pre-publication history for this paper can be accessed here:

http://www.biomedcentral.com/1471-2490/4/5/prepub
Publish with Bio Med Central and every scientist can read your work free of charge

"BioMed Central will be the most significant development for disseminating the results of biomedical research in our lifetime. " Sir Paul Nurse, Cancer Research UK

Your research papers will be:

- available free of charge to the entire biomedical community

- peer reviewed and published immediately upon acceptance

- cited in PubMed and archived on PubMed Central

- yours - you keep the copyright

Submit your manuscript here:

http://www.biomedcentral.com/info/publishing_adv.asp 